\title{
Sorting-out of acceptor-donor relationships in the transglutaminase- catalyzed cross-linking of crystallins by the enzyme-directed labeling of potential sites
}

\author{
L. Lorand*, K. N. Parameswaran, and P. T. Velasco \\ Department of Biochemistry, Molecular Biology, and Cell Biology, Northwestern University, Evanston, IL 60208
}

Contributed by Laszlo Lorand, October 8, 1990

\begin{abstract}
The dansyl-conjugated (Dns) peptides DnsPro-Gly-Gly-Gln-Gln-Ile-Val and Dns-Ala-Gln-Gln-Ile-Val, patterned on the $\mathbf{N}$-terminal sequence of fibronectin, were synthesized and used for the transglutaminase (proteinglutamine;amine $\boldsymbol{\gamma}$-glutamyltransferase, EC 2.3.2.13)-directed selective blocking of lens proteins that otherwise might participate in donating lysyl side chains in forming $N^{\varepsilon}$-( $\gamma$-glutamyl)lysine cross-linked oligomers and polymers. Labeling profiles with these peptides could be readily visualized by fluorescence as well as by immunoblotting with anti-dansyl antibody. The labeling patterns in rabbit lens homogenates were quite different with the dansylated peptides than those obtained with dansylcadaverine. Use of such glutamine-containing dansylated peptides should clearly aid in identifying, isolating, and sequencing potential donor substrates of transglutaminases in many biological systems.
\end{abstract}

The success of inhibiting cross-linking reactions mediated by transglutaminase (protein-glutamine:amine $\gamma$-glutamyltransferase, EC 2.3.2.13) by specifically blocking donor functionalities with glutamine-containing peptide analogues of the $\mathrm{N}$-terminal sequence of fibronectin (1) spurred the current efforts to synthesize similarly acting compounds with readily recognizable reporter groups such as 5-dimethylaminonaphthalene-1-sulfonyl (dansyl) or biotin. First we focused on dansylated derivatives because, in addition to fluorescence detection, methodologies are already in place for immunoblotting and also for the eventual isolation of the tracercarrying sequences with anti-dansyl antibodies $(2,3)$. The present report describes our findings with two dansylconjugated (Dns) peptides, Dns-Pro-Gly-Gly-Gln-Gln-IleVal and Dns-Ala-Gln-Gln-Ile-Val, and demonstrates their usefulness in sorting out the potential acceptor and donor subunits for the cross-linking of crystallins catalyzed by the intrinsic transglutaminase in lens homogenates.

\section{MATERIALS AND METHODS}

Dansylated Peptides. Dns-Ala-Gln-GIn-Ile-Val was obtained by treatment of the benzyl ester of Ala-Gln-Gln-Ile-Val (1) with dansyl chloride (Aldrich) in dimethylformamide in the presence of triethylamine, followed by removal of the ester by catalytic hydrogenation. Dns-Pro-Gly-Gly-Gln-GlnIle-Val was synthesized by coupling of the tripeptide DnsPro-Gly-Gly to the benzyl ester of Gln-Gln-Ile-Val (1), followed by hydrogenation.

Inhibition of Crystallin Cross-Linking in Lens Homogenate. Frozen lenses from young rabbits (Pel-Freez Biologicals) were thawed and decapsulated, and the cortex was separated from the nucleus. The cortical portions from three lenses were homogenized in $2 \mathrm{ml}$ of $50 \mathrm{mM}$ Tris $\cdot \mathrm{HCl}, \mathrm{pH} \mathrm{7.5/100}$

The publication costs of this article were defrayed in part by page charge payment. This article must therefore be hereby marked "advertisement" in accordance with 18 U.S.C. $\$ 1734$ solely to indicate this fact.
$\mathrm{mM} \mathrm{NaCl}$ by hand in a Potter-Elvehjem tissue grinder. Incubations were carried out at $37^{\circ} \mathrm{C}$ in a total volume of 100 $\mu l$ containing homogenate (approximately $50 \mathrm{mg}$ of protein per $\mathrm{ml}$ ), $20 \%$ (vol/vol) glycerol, $2 \mathrm{mM}$ leupeptin (obtained through the U.S.-Japan Cooperative Cancer Research Program), $2 \mathrm{mM}$ of one of the dansylated peptides or dansylcadaverine (cadaverine is 1,5-diaminopentane), and either 8 $\mathrm{mM} \mathrm{CaCl} 2$ or $2 \mathrm{mM}$ EDTA. Mixtures were incubated for 15 min at $37^{\circ} \mathrm{C}$ prior to the addition of one of the latter two components. The reactions were then allowed to proceed at the same temperature for a period of $90 \mathrm{~min}$, when they were stopped by the addition of $20 \mu \mathrm{l}$ of $100 \mathrm{mM}$ EDTA. The samples were centrifuged $(15,600 \times g$ for $5 \mathrm{~min})$, and $10 \mu \mathrm{l}$ of the supernatants were incubated with $100 \mu$ l of $50 \mathrm{mM}$ Tris $\cdot \mathrm{HCl}, \mathrm{pH} 7.1 / 9 \mathrm{M}$ urea/2\% sodium dodecyl sulfate (SDS)/40 mM dithiothreitol for $60 \mathrm{~min}$. Approximately 130 $\mu \mathrm{g}$ of proteins were analyzed by SDS/PAGE using the discontinuous buffer system of Laemmli (4) on 1.5-mm-thick gels in a protean slab gel apparatus (Bio-Rad). A stacking gel of $3 \%$ acrylamide, a resolving gel of $12 \%$ acrylamide, and a running buffer of $25 \mathrm{mM}$ Tris/192 mM glycine/0.1\% SDS, $\mathrm{pH}$ 8.6, were used. The gel was stained with Coomassie brilliant blue $\mathrm{R}$ and was calibrated with molecular weight standards (Bio-Rad) of phosphorylase b $\left(M_{\mathrm{r}} 97,400\right)$, bovine serum albumin $\left(M_{\mathrm{r}} 66,200\right)$, ovalbumin $\left(M_{\mathrm{r}} 45,000\right)$, carbonic anhydrase $\left(M_{\mathrm{r}} 31,000\right)$, soybean trypsin inhibitor $\left(M_{\mathrm{r}} 21,500\right)$, and lysozyme $\left(M_{\mathrm{r}} 14,400\right)$. Electroblotting $(75 \mu \mathrm{g}$ per lane) to nitrocellulose $(0.2 \mu \mathrm{m}$; Schleicher and Schuell) was carried out with a LKB model 2005 electroblotting unit for $2 \mathrm{hr}$ at $4^{\circ} \mathrm{C}$ by the method of Towbin et al. (5) in $25 \mathrm{mM}$ Tris/192 mM glycine, $\mathrm{pH} 8.3 / 20 \%$ (vol/vol) methanol. For purposes of immunostaining, a rabbit IgG to dansylated bovine gamma globulins was diluted $1: 5000$ into $10 \mathrm{mM}$ sodium phosphate, pH 7.1/0.9\% NaCl/0.05\% Tween 20 and a Vectastain ABC kit (Vector Laboratories) was used for the peroxidase-based staining (6).

\section{RESULTS AND DISCUSSION}

The cross-linking reaction in rabbit lens homogenate, as catalyzed by the intrinsic transglutaminase upon addition of $\mathrm{Ca}^{2+}$, is of particular interest because-among all the cytoplasmic proteins present-only a small population of $\beta$ crystallin subunits are involved. None of the $\alpha$ or $\gamma$ crystallins seems to participate directly in forming $N^{\varepsilon}$-( $\gamma$-glutamyl $)$ lysine-linked structures $(7,8)$. Kinetically, the first crosslinked products are the dimeric $\beta$ crystallins and these were followed by formation of higher ordered oligomeric and polymeric structures. Protein profiles on SDS/PAGE suggest that generation of dimeric $\beta$ crystallins is not a homologous fusion of two identical $\beta$ crystallin subunits.

Abbreviation: Dns, dansyl conjugated to peptides.

*To whom reprint requests should be addressed. 
Previously with the use of dansylcadaverine, while blocking the formation of dimeric $\beta$ crystallins, we were able to label only the $\beta$ crystallin subunits that acted as the potential amine acceptor partners in the transglutaminase-mediated reaction. These effects of dansylcadaverine are also demonstrated in Fig. 1C. The recent observation that certain endo-glutamine-containing peptides, patterned on the N-terminal sequence of fibronectin, could inhibit cross-linking in the lens system while selectively modifying some crystallins (1) offered the opportunity to identify the potential amino group-donating protein subunits.

Sorting-out Acceptor-Donor Relationships in the Transglutaminase-Catalyzed Cross-Linking in Lens

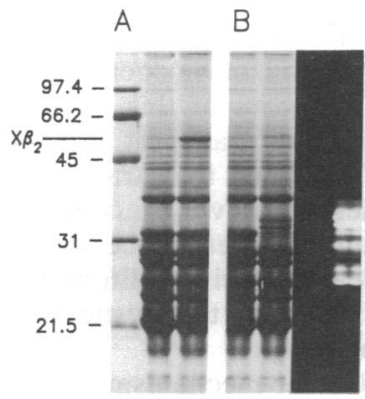

12

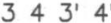

$565^{\prime} 6$

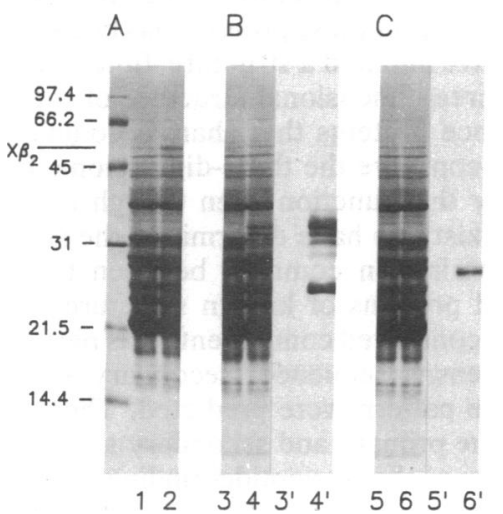

FIG. 1. Inhibition of crystallin cross-linking in lens homogenate by Dns-Pro-Gly-Gly-Gln-Gln-Ile-Val or dansylcadaverine. For the method of activating the intrinsic transglutaminase in rabbit lens homogenate, see Materials and Methods. Samples contained no inhibitor $(A), 2 \mathrm{mM}$ Dns-Pro-Gly-Gly-Gln-Gln-Ile-Val $(B)$, or $2 \mathrm{mM}$ dansylcadaverine $(C)$ and either $10 \mathrm{mM} \mathrm{CaCl}_{2}$ (lanes 1,3 , and 5) or 2 mM EDTA (lanes 2, 4, and 6) . (Upper) Following SDS/PAGE, the gel was photographed under UV light (366 nm; lanes $\left.3^{\prime}-6^{\prime}\right)$ and stained with Coomassie brilliant blue $\mathrm{R}$ (lanes 1-6). (Lower) Alternatively, the gel was electroblotted and the nitrocellulose sheets were either stained with Amidoblack (lanes 1-6) or immunostained with anti-dansyl IgG (lanes $3^{\prime}-6^{\prime}$ ). The fluorescence on the gels with dansylcadaverine revealed that the band Upper $C$, lane $6^{\prime}$, was in fact a doublet. Cross-linked dimeric $\beta$ crystallins are marked $X \beta_{2}$.
Given the analytical advantages afforded by a dansyl probe, we prepared two peptides carrying the fluorescent and haptenic moiety: Dns-Pro-Gly-Gly-GIn-Gln-Ile-Val and DnsAla-Gln-Gln-Ile-Val. Fig. $1 B$ presents our findings with the former compound; results with the other derivative were quite similar, though the impression was gained that inhibition of cross-linking was somewhat better with Dns-Pro-GlyGly-Gln-Ile-Val. Formation of dimeric $\beta$ crystallins could, in fact, be inhibited by as little as $0.5 \mathrm{mM}$ of this compound.

The most important observation was that the transglutaminase-mediated labeling pattern of proteins with dansylcadaverine was quite different from that obtained with the glutamine-containing dansylated peptides. This is proved by fluorescence detection (Fig. 1 Upper) as well as anti-dansyl antibody-dependent immunostaining (Fig. 1 Lower). The obvious conclusion is that dansylcadaverine visualizes the potential acceptor sites, whereas the dansylated peptides specifically mark the donor proteins that could conceivably contribute the lysyl side chains to the $N^{\varepsilon}$-( $\gamma$-glutamyl)lysine bridges in heterologous cross-linking.

As previously noted (1), peptide-decorated crystallins undergo an upward shift in SDS/PAGE to positions of apparent higher molecular weight values depending on the number of transglutaminase-reactive donor groups actually blocked, and this was also seen with the dansylated compounds. Hence, some of the multiplets visualized in lanes $4^{\prime}$ of Fig. $1 B$ could represent different degrees of modifications of the same protein subunit. Nevertheless, a comparison of the labeling profiles with the glutamine-containing dansylated peptides and dansylcadaverine (in Fig. 1, compare lanes $4^{\prime}$ in $B$ with lanes $6^{\prime}$ in $C$ ) suggests that the acceptor subunits, marked with the latter probe, rather than donors may be the limiting component for generating dimeric $\beta$ crystallins.

Use of such glutamine-containing dansylated peptides in an analogous manner will undoubtedly also aid in identifying, isolating, and sequencing potential donor substrates of transglutaminases in other biological systems.

This work was aided by Public Health Service Research Career Award (HL03512) and by a grant from the National Institutes of Health (EY03942).

1. Parameswaran, K. N., Velasco, P. T., Wilson, J. \& Lorand, L. (1990) Proc. Natl. Acad. Sci. USA 87, 8472-8475.

2. Cariello, L., Velasco, P. T., Wilson, J., Parameswaran, K. N., Karush, F. \& Lorand, L. (1990) Biochemistry 29, 5103-5108.

3. Velasco, P. T. \& Lorand, L. (1988) Invest. Ophthalmol. Vis. Sci. 29, 184 (abstr.)

4. Laemmli, U. K. (1970) Nature (London) 227, 680-685.

5. Towbin, H., Staehelin, T. \& Gordon, J. (1979) Proc. Natl. Acad. Sci. USA 76, 4350-4354.

6. Lorand, L., Murthy, S. N. P., Velasco, P. T. \& Karush, F. (1986) Biochem. Biophys. Res. Commun. 134, 685-689.

7. Lorand, L., Conrad, S. M. \& Velasco, P. T. (1985) Biochemistry 24, 1525-1531.

8. Velasco, P. T. \& Lorand, L. (1987) Biochemistry 26, 4629-4634. 